\title{
Carbonic anhydrase inhibition as a cancer therapy: a review of patent
} literature 2007-2009

\begin{abstract}
Importance of the field: Despite advances in cancer therapeutics, treatment options are still limited for many types of cancer. The development of new treatments that are curative and allow physicians to maintain quality of life for patients, while selectively attacking the cancer tissue are desperately needed. One approach towards this goal is to understand what distinguishes cancer cells from normal cells so that rational, mechanism-based molecular therapeutics for cancer may be developed. The functional contribution of membrane-bound extracellular carbonic anhydrases (CAs) to hypoxic tumor growth and progression has long been hypothesized; however recent convergent evidence from a number of groups strongly implicates these CAs as key prosurvival enzymes during tumour hypoxia. From this perspective targeting the inhibition of cancer-associated CA enzymes, most notably CA IX and XII, has recently been identified as a mechanistically novel scientific opportunity with great potential as a new cancer drug target.
\end{abstract}

Areas covered in this review: This review covers world patent applications filed during the period 2007 until 2009. These applications describe the modulation of CA activity as a treatment approach for hypoxic tumors and encompass (i) classical small molecule chemotherapies; (ii) small molecule radiopharmaceuticals; (iii) biologics such as antibodies as both chemo- and immunotherapies; and (iv) indirect approaches, for example the inhibition of CA gene expression. This review covers only small molecule approaches of CA cancerrelated patents (i) and (ii), while the non-small molecule approaches are not within the scope of this review. 
What the reader will gain: The reader will be provided with a background of the biology of CAs as well as the recent research findings that have validated the crucial pro-survival role of CAs in hypoxic tumors. The review will highlight small molecule molecular methods that modulate CAs as an anti-cancer therapeutic strategy.

Take home message: Our present understanding of the pivotal role of CAs in tumor hypoxia is related to results of very recent studies, so that while therapeutic applications of CA inhibitors have been known for many decades (as reviewed previously in this journal) ${ }^{1}$ the potential of CAs as a therapeutic target for oncology is a relatively fresh therapeutic application. Much of what has been reported in the patent literature during the period 20072009 is based on alleged therapeutic benefits of CA inhibitors in cancer. The field has lacked appropriate CA-relevant cell and animal models of tumor hypoxia for the evaluation of compounds. These models have however become available recently, and although not yet in widespread use, this deficiency should be addressed in the short to medium term. The take home message is to 'watch this space'. The verification of the ability of small molecules to modulate CA activity as a cancer therapy or as a diagnostic and/or prognostic tool is now possible and probable. The CA field will thus provide for a scientifically exciting and possibly rewarding next few years, accelerated by the growing interest in the potential clinical applications of this enzyme class in oncology.

\section{Keywords}

cancer; carbonic anhydrase; carbonic anhydrase inhibitor; click chemistry; hypoxia; nitric oxide; radiopharmaceutical; sulfamate; sulfonamide 


\section{Introduction}

1.1 Background: Carbonic anhydrase enzyme activity. Carbonic anhydrases (CAs, EC 4.2.1.1) are zinc metalloenzymes that catalyze the reversible hydration of carbon dioxide $\left(\mathrm{CO}_{2}\right)$ to generate bicarbonate anion $\left(\mathrm{HCO}_{3}^{-}\right)$and a proton $\left(\mathrm{H}^{+}\right)$, Scheme $1 .{ }^{2}$ The substrates of the CA catalyzed reaction are known to regulate a broad range of physiological functions that involve the production and transport of carbon dioxide, protons and bicarbonate. This equilibrium underpins respiration and transport of $\mathrm{CO}_{2}$ and $\mathrm{HCO}_{3}^{-}$between metabolizing tissues and lungs, $\mathrm{pH}$ homeostasis, bone development and function, electrolyte secretion, biosynthesis where $\mathrm{HCO}_{3}^{-}$is a substrate, among others. ${ }^{2,3}$ The hydration of $\mathrm{CO}_{2}$ under physiological conditions, although spontaneous, does not proceed fast enough in the absence of CAs to support these biological processes. ${ }^{2}$

Scheme 1. Carbonic anhydrases catalyze the reversible hydration of carbon dioxide to give bicarbonate anion and a proton.

$$
\mathrm{CO}_{2}+\mathrm{H}_{2} \mathrm{O} \leftrightarrows \mathrm{HCO}_{3}^{-}+\mathrm{H}^{+}
$$

The clinical modulation of CA activity by small molecule inhibitors has proven a reliable treatment for a range of human disease states and has been a mainstay of antihypertensive, antiglaucoma, antithyroid and hypoglycemic drug treatment regimes for several decades. ${ }^{3}$ At least 25 clinically used drugs have been reported to possess significant CA inhibitory properties. $^{3}$ There are 12 catalytically active human CA isozymes that have been characterized. The catalytic domains of these enzymes contain a conserved active site $\mathrm{Zn}^{2+}$ that is coordinated to three histidine amino acid sidechains. This $\mathrm{Zn}^{2+}$ is a strong Lewis acid and binds to and activates a substrate $\mathrm{H}_{2} \mathrm{O}$ molecule as the fourth ligand. The $\mathrm{Zn}^{2+}$ coordinated 
$\mathrm{H}_{2} \mathrm{O}$ molecule has a $\mathrm{p} K_{\mathrm{a}}$ of $\sim 7$ compared to the $\mathrm{p} K_{\mathrm{a}}$ of bulk water $\sim 14{ }^{2}$ CA thus facilitates the deprotonation of $\mathrm{H}_{2} \mathrm{O}$ to generate the strongly basic hydroxide at physiological $\mathrm{pH}$. Hydroxide is the reactive species in the hydration of $\mathrm{CO}_{2}$ leading to formation and release of $\mathrm{HCO}_{3}{ }^{-}$, Scheme 2 . $^{2}$

Scheme 2. Catalytic cycle of CAs. The active site $\mathrm{Zn}^{2+}$ is coordinated to three histidine residues and the substrate $\mathrm{H}_{2} \mathrm{O}$ molecule. The $\mathrm{Zn}^{2+}$ coordinated water is deprotonated to generate the strongly basic hydroxide anion which is the reactive species in the hydration of $\mathrm{CO}_{2}$ converting it to $\mathrm{HCO}_{3}^{-}$.

1.2 Carbonic anhydrases and tumor hypoxia. In solid tumors vasculature is poor and limits the blood supply to the tumor mass, this in turn reduces the delivery of $\mathrm{O}_{2}$ to tumor cells. Low $\mathrm{O}_{2}$, or hypoxia, induces the activation of the hypoxia-inducible transcription factor (HIF). ${ }^{4}$ High levels of HIF regulate a signalling cascade involving 100 genes that initiate adaptive cellular functions to allow solid tumor cells to survive hypoxia, to proliferate and to metastasize. ${ }^{4}$ CA IX is one of the most highly induced HIF responsive genes and is overexpressed and sustained in a large number of solid tumors including breast, brain (glioblastoma), clear cell renal, colorectal, head and neck, bladder and non-small cell lung carcinomas, ${ }^{5-7}$ yet CA IX has limited distribution and expression in normal tissues. ${ }^{5-7}$ CA IX 
is a multidomain protein consisting of an N-terminal proteoglycan like domain, a transmembrane domain, a short intracellular domain and an extracellular catalytic domain. ${ }^{8}$

Tumor cells experience elevated metabolism and thus increased acid production compared to healthy cells. ${ }^{4,9,10}$ For some time it was thought that tumor cells had both a lower intracellular $\mathrm{pH}\left(\mathrm{pH}_{\mathrm{i}}\right)$ and extracellular $\mathrm{pH}\left(\mathrm{pH}_{\mathrm{e}}\right)$, however it became apparent from a number of studies that tumor cells actually maintain a normal $\mathrm{pH}_{\mathrm{i}} \cdot{ }^{10}$ Tumour cells have evolved several mechanisms to extrude acid into the extracellular environment to counteract the acid load of elevated metabolism and these mechanisms contribute to lowering extracellular $\mathrm{pH}\left(\mathrm{pH}_{\mathrm{e}}\right)^{10}$ which favours tumor invasive behaviour and development. ${ }^{11}$ Recent evidence has demonstrated that cell-generated $\mathrm{CO}_{2}$ in addition to lactic acid is responsible for the rapid removal of acid equivalents from tumor cells. ${ }^{9,12,13}$ Cell-derived $\mathrm{CO}_{2}$ is membrane permeable, so provided there is sufficient outward gradient this $\mathrm{CO}_{2}$ diffuses to the extracellular space, where in poorly vascularised hypoxic tumor tissue, it is hydrated to $\mathrm{HCO}_{3}{ }^{-}$and a $\mathrm{H}^{+}$by CA IX (and/or CA XII). ${ }^{9,12,13}$ The functional contribution of CAs to hypoxic tumor growth and progression has long been hypothesized; however recent convergent evidence from a number of groups strongly implicates these extracellular membrane-bound CAs are the key regulatory molecules for countering acidosis during hypoxia, Figure 1. ${ }^{9,12-14}$ The net effect of CA IX/XII is to trap acid extracellularly, lowering extracellular $\mathrm{pH}\left(\mathrm{pH}_{\mathrm{e}} \sim 6.9-7.0\right)$ and maintaining normal $\mathrm{pH}_{\mathrm{i}}(\sim 7.2)$ with $\mathrm{HCO}_{3}{ }^{-}$recycled back into the cell. ${ }^{9,12,13}$

Figure 1. Schematic of normoxic and hypoxic cells demonstrating the pro-survival role of CAs in hypoxic tumours through $\mathrm{pH}_{\mathrm{i}}$ regulation. 
1.3 Recent progress in the carbonic anhydrase field. G250 is a monoclonal antibody that underwent PhaseI/II clinical trials as a radioimmunotherapy for renal cancer long before its antigen was identified as CA IX in 2000. ${ }^{15,16}$ This vital connection between G250 and extracellular CA IX has triggered a rigorous research effort into the role of CAs in cancer. Early in 2009 a thorough in vivo study of the role of CA IX and XII using shRNA gene silencing technology in a transfected LS174T colorectal cancer xenograft models was reported. ${ }^{9}$ In this study the ca9 gene was silenced and caused tumor regression with a $40 \%$ reduction in xenograft tumor volume reported. A further new finding was that ca12 gene expression was elevated following ca9 gene silencing. When both ca9 and ca12 gene expression were silenced a dramatic $85 \%$ reduction in tumor volume was observed. Also highly significant was that silencing of HIF expression was much less effective than the combined silencing of the ca9 and ca12 genes. This study provided definitive evidence that CA IX and XII confer on hypoxic tumor cells a survival and growth advantage in an acidic microenvironment through $\mathrm{pH}_{\mathrm{i}}$ maintenance and regulation. The results from targeting CA IX with antibodies and CA IX and XII with gene silencing has significantly advanced our understanding of the pivotal role of CAs in hypoxic tumor cell survival. These findings have validated the long held hypothesis that CA IX and CA XII constitute new anticancer 
therapeutic targets with a novel mechanism of action and strongly suggest that CA is worthy of scrutiny as a potential new cancer therapy. ${ }^{9,13}$

1.4 Carbonic anhydrase inhibitors. The classical small molecule CA inhibitors that bind the active site of CAs are aromatic or heteroaromatic sulfonamides $\left(\mathrm{R}^{-} \mathrm{SO}_{2} \mathrm{NH}_{2}\right){ }^{2,3}$ The sulfonamide anion ( $\mathrm{R}-\mathrm{SO}_{2} \mathrm{NH}^{-}$) coordinates to the $\mathrm{CA}$ active site $\mathrm{Zn}^{2+}$ and resembles the structure of CA with native substrates, Figure 2., ${ }^{2,3}$ As a transition state analogue, this R$\mathrm{SO}_{2} \mathrm{NH}_{2}$ motif has given rise to many very effective $\mathrm{CA}$ inhibitors. Clinically used $\mathrm{CA}$ inhibitors include acetazolamide, ethoxazolamide, brinzolamide and dichlorophenamide, Figure $3 .^{3}$ As the active site of human CAs are structurally very similar, CAs are a challenging drug target for developing isozyme selective inhibitors. As targets however, the HIF induced CA isozymes possess two discerning attributes that provide opportunities for selective targeting with small molecule inhibitors. First, hypoxic tumors overexpress CA IX and XII while many healthy tissues lack these isozymes. ${ }^{5,6}$ Second, unlike the cytosolic CA I and II (off-target), CA IX and XII are transmembrane proteins with an extracellular enzyme active site. The properties of small molecules may be modified to take advantage of the lipid bilayer membrane as a 'selectivity barrier' between off-target CAs and cancer-associated CAs. ${ }^{17-22}$

Figure 2. Bicarbonate substrate and sulfonamide inhibitor $\left(\mathrm{R}-\mathrm{SO}_{2} \mathrm{NH}_{2}\right)$ bound in the active site of CAs. 
Figure 3. Clinically used aromatic or heteroaromatic sulfonamide inhibitors of CAs. All are representatives of the $\mathrm{R}-\mathrm{SO}_{2} \mathrm{NH}_{2}$ pharmacophore.

\section{Discussion of selected world patent applications.}

\subsection{Nitrate esters of CA inhibitors}

NiCox S.A. is a pharmaceutical company engaged in the development of nitric oxide (NO) donating drugs. NiCox S.A. has demonstrated an extended interest in CA inhibition with the filing of an international patent application describing small molecule compounds that contain both a sulfonamide moiety for CA inhibition and a nitrate ester to release NO. ${ }^{23}$ The application proposes these dual action compounds for use as therapeutic treatments against a long list of health disorders related to CA inhibition, this includes eye disorders (glaucoma, ocular hypertension, age-related macular degeneration, diabetic macular edema, diabetic retinopathy and retinal vasculopathies), cancer, epilepsy, high-altitude disorders and neuromuscular diseases. The premise behind the combination of CA inhibitions with NO release is to provide therapeutic advantages over CA inhibition alone. The application presents the synthesis and structures for 208 compounds. Of these, 123 compounds contain a primary sulfonamide moiety needed for CA inhibition. The remaining 85 compounds contain a secondary sulfonamide, and unless these are prodrugs of a primary sulfonamide they are unlikely to be CA inhibitors. All compounds contain the nitrate group for NO release. The patent application states that the compounds have improved pharmacological profiles 
compared to existing clinically used CA inhibitors, including enhanced tolerability and long acting ocular hypotensive activity, however no pharmacological data to support these statements is provided within the application.

The application reports CA inhibition for six of the 208 compounds, specifically inhibition of hCA I, II, IV, IX and XII (where $\mathrm{h}=$ human). Inhibition was determined using the $\mathrm{CO}_{2}$ hydration assay, a stopped flow technique that monitors the physiological reaction catalyzed by CAs, i.e. $\mathrm{CO}_{2}$ hydration to bicarbonate anions and protons. Of particular interest for cancer therapy applications is the CA IX and XII inhibition data as well as off-target CA inhibition characteristics. For the six compounds the $\mathrm{IC}_{50}$ s range from 20-355 nM for CA IX, 28-86 nM for CA XII and 10-67 nM for CA II, hence the compounds were not selective for the active site of cancer-associated CAs over CA II. The application next describes the in vitro evaluation of compounds to induce vasorelaxation in an isolated rabbit thoracic aorta preparation. All of the nitrate esters were reported to have $\mathrm{EC}_{50}$ values in the range 1-50 $\mu \mathrm{M}$ 
in this assay, where the $\mathrm{EC}_{50}$ represents the concentration of test compound needed to produce $50 \%$ of the maximum relaxation. A comparison with 'native' CA inhibitors (i.e. those without NO releasing capacity) was discussed, with the native CA inhibitors producing no vasorelaxation effect. The chemical names, chemical structures nor number of 'native’ CA inhibitors evaluated were disclosed in the application. With regards to the claims that these compounds may be of therapeutic benefit for treatment of cancer, there is neither in vitro nor in vivo pharmacological evidence within the application to support these claims. The therapeutic effects for cancer are alleged on the basis of CA IX and XII enzyme inhibition data.

\subsection{Sulfamate CA inhibitors}

Solvay Pharmaceuticals GMBH filed an international patent application describing sulfamate compounds for medical use (treatment and/or prophylaxis) for a very broad range of health disorders, including obesity, diabetes mellitus type I and II, metabolic syndrome X, diabetic neuropathy, diabetic retinopathy, diabetic nephropathy, diabetic microangiopathy, diabetic macroangiopathy, insulinoma, familial hyperinsulemic hypoglycemia, male pattern baldness, detrusor hyperreactivity, hypertension, asthma, glucose metabolism disorders, Parkinson’s disease, Alzheimer's disease, arrhythmia, impotence, glaucoma, dipolar disorders, migraine, cardiovascular disease and cancer. ${ }^{24}$ Sulfamates are isosteres of sulfonamides, wherein the sulfonamide sulphur atom is attached to an electronegative oxygen atom with the general formula R-O-SO ${ }_{2} \mathrm{NH}_{2}$ and can bind to the active site $\mathrm{Zn}^{2+}$ similarly to Figure 2. Topiramate, a fructopyranose sulfamate, is a billion dollar drug that is marketed worldwide for the treatment of epilepsy and migraine. ${ }^{25}$ This compound has been shown to be a good inhibitor of CAs in vitro by the $\mathrm{CO}_{2}$ hydration assay. ${ }^{26}$ Since this finding a number of sulfamates with excellent CA inhibition have been reported in the literature. ${ }^{27}$ 
This patent application describes the synthesis of sulfamates of the general structure $\mathbf{1 2 4}$ (for CA inhibition $\mathrm{R}^{1}=\mathrm{R}^{2}=\mathrm{H}$ ). Compounds contain one or more asymmetric centres, and a representative structure from this application is compound 125. The sulfamates are synthesized by the reaction of a primary alcohol with sulfamoyl chloride, this being well established chemistry. ${ }^{28}$ The compounds of this invention are reported to have CA I, II and VB inhibition activity, with inhibition determined using a fluorescence based assay in which compounds are assessed for their ability to displace the fluorescent sulfonamide ligand, 5(dimethylamino)-1-naphthalenesulfonamide (DNSA), from the CA active site. The \% inhibition data for six different sulfamates at a single point test compound concentration; either 0.1 or $1 \mu \mathrm{M}$ for CA I and II or $10 \mu \mathrm{M}$ for CA VB were reported. The inhibition varied from $15-85 \%$.

The application describes a number of biological studies associated with the therapeutic claims. These include (i) an acute in vivo food intake test in mice (inhibitors reduce food intake and have potential anti-obesity properties), (ii) the effect on neurite outgrowth in hippocampal neurons using a cell based assay (inhibitors promote growth and have potential value in neurodegenerative diseases), (iii) an electroconvulsive shock threshold test to evaluate anticonvulsant properties in a mouse (inhibitors are anticonvulsive and have potential antiepileptic properties), (iv) a diabetic induced neuropathic pain test in a rat diabetes model (inhibitors provided neuropathic pain relief). The application does not present 
biological data of relevance to the cancer therapy claims. CA inhibition data for the cancerassociated CA isozymes IX and XII is not provided or discussed, nor are results from cancer cell-based assays or animal models of cancer.

\subsection{Benzimidazo[1,2,-c][1,2,3]thiadiazol-7-sulfonamides as CA inhibitors.}

A patent application lodged by the Institute of Biotechnology, Lithuania, describes the synthesis a series of tricyclic primary sulfonamides as CA inhibitors. ${ }^{29}$ Compounds of the general structure $\mathbf{1 2 6}$ are described, wherein the primary sulfonamide group resides in all four possible positions on the benzene ring. Benzimidazo[1,2,-c][1,2,3]thiadiazol-7-sulfonamides have been reported in the literature by the applicants ${ }^{30}$ as CA inhibitors prior to this patent application, while the 5-, 6- and 8-sulfonamide derivatives represent new and novel structures. The application provides descriptions of the synthesis of both the intermediates and final target compounds. The application claims potential biomedical applications of the compounds, which are stated to be nontoxic and without side effects associated with inhibition of off-target CA isozymes.

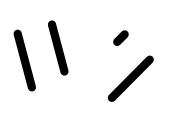

The binding of the tricyclic sulfonamides type 126 to hCA I and bCA II (b = bovine) was measured using two different assay approaches: an isothermal titration calorimetry (ITC) assay and a fluorescent thermal shift assay. Using the ITC assay method the observed binding constants for all tested tricyclic sulfonamides were reported as $10^{6}-10^{7} \mathrm{M}^{-1}$, while the intrinsic binding constants were presented as better CA binders than standard CA inhibitors 
ethoxazolamide, acetazolamide, trifluoromethane sulfonamide and pentafluorobenzene sulfonamide. A qualitative interpretation of experimental results was presented for three tricyclic sulfonamide compounds together with the four standard CA inhibitors. A fluorescent thermal shift assay method to evaluate CA inhibitor binding was briefly described. With this method the inhibitor induced CA enzyme thermal stabilization is monitored in the presence of 1-anilino-8-napthalene sulfonate. The observed binding constant data determined using this method was qualitatively reported as consistent with the ITC method.

This patent application claims the compounds of type $\mathbf{1 2 6}$ have improved CA isozyme selectivity than the standard CA inhibitors. The validity of this claim in the absence of inhibition data against all catalytically active human CA isozymes is questionable. Of particular relevance to this review is that studies of the compounds with the cancer-associated CA isozymes were neither presented nor discussed.

\subsection{CA IX inhibitors as radiopharmaceuticals -1 .}

Siemens Medical Solutions USA, Inc. has filed a world patent application describing the parallel synthesis of CA inhibitors using click chemistry, and the subsequent synthesis and use of selected inhibitors as positron emission tomography (PET) imaging agents. ${ }^{31}$ The application is based on the use of CA IX inhibitors in PET to identify and monitor CA IX expression in vivo and to predict the likely efficacy of CA IX inhibitors administered as a therapy to treat disease associated with the overexpression of CA IX, such as hypoxic cancers. The background to this application is the association of CA IX expression with hypoxic tumors and hence the biomarker potential of this CA isozyme in cancer diagnostics. Highlighted as of particular interest were cancers of the breast, gastrointestinal tract, stomach 
(including esophagus), colon, kidney, prostate, liver, urinary tract (including bladder), lungs, head and neck, and gynaecological cancers.

The concept of the 'tail approach' for developing selective CA inhibitors was first described by Supuran and co-workers. ${ }^{32}$ Since this initial report the linking of an aromatic or heteroaromatic sulfonamide scaffold to a 'tail' group has proven a reliable means to alter the physicochemical properties of CA inhibitors. The linking of the 'tail' moiety has been achieved using myriad synthetic chemistry reactions including click chemistry, cross metathesis, amide, ester, urea, thiourea, sulfonamide etc. ${ }^{17,33-35}$ This patent application describes the $\mathrm{Cu}(\mathrm{I})$ catalyzed 1,3-dipolar cycloaddition reaction of organic azides with terminal alkyne moieties (i.e. click chemistry) for the formation of a 1,2,3-triazole as the covalent linkage. This "click tailing” approach has been described by others previously. ${ }^{33}$ Compounds 127-129 represent exemplary CA inhibition scaffolds from this application. These scaffolds have both the primary sulfonamide moiety needed for CA inhibition and the alkyne moiety needed to participate in click chemistry.

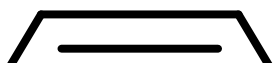

Compounds 127-129 were initially utilized to generate three combinatorial libraries of CA inhibitors by reaction with a large panel of azides $\left(\mathrm{R}-\mathrm{N}_{3}\right)$ in parallel, Scheme 3 . Following this the libraries were elaborated to generate further 1,2,3-triazole CA inhibitors.

Scheme 3. Synthesis by $\mathrm{Cu}(\mathrm{I})$ catalyzed 1,3-dipolar cycloaddition reaction of azide with alkyne CA inhibitor scaffold 127. 
All 1,2,3-triazole CA inhibitors were evaluated at a test compound concentration of $1 \mu \mathrm{M}$ for CA II and CA IX binding using the fluorescence based assay with DNSA as a competitive CA binder. Compounds identified with a good CA binding were followed up to determine dissociation constants or $K_{d} s$. A number of compounds with $K_{d}$ values in the low $n M$ range at both CA IX and II where presented. The compounds for which results were presented were typically 10-fold more potent binders at CA II than at CA IX. The patent application reports tabulated assay results data for a large number of compounds. Further to CA binding, a red blood cell membrane permeability assay was conducted for selected inhibitors. The significance of this assay is that CA inhibitors that lack red blood cell permeability are less likely to have off-target CA inhibition side effects as red blood cells contain high levels of CA II. The majority of compounds presented were cell permeable to an extent similar to the standard CA inhibitor ethoxazolamide, used as a control. There were however several compounds with limited permeability identified, the structures of these membrane impermeant compounds were not revealed in the application but these are likely to be valuable lead compounds for the development of radiopharmaceutical applications.

The typical synthesis of a radiolabelled analogue (for example 134) for PET applications is shown in Scheme 4. The primary sulfonamide group is first protected (eg. with a dimethoxytrityl (DMTr) group), followed by the nucleophilic displacement of a leaving group 
(eg. nosylate or tosylate) elsewhere in the CA inhibitor molecule with a radionuclide such as $\left[{ }^{18} \mathrm{~F}\right]$ fluoride anion. The final step is the deprotection of the sulfonamide moiety.

Scheme 4. Synthesis of a triazole CA inhibitor (compound 134) as a PET ligands for imaging CA IX expressing tumors. $\mathrm{P}=$ protecting group, $\mathrm{LG}=$ leaving group.
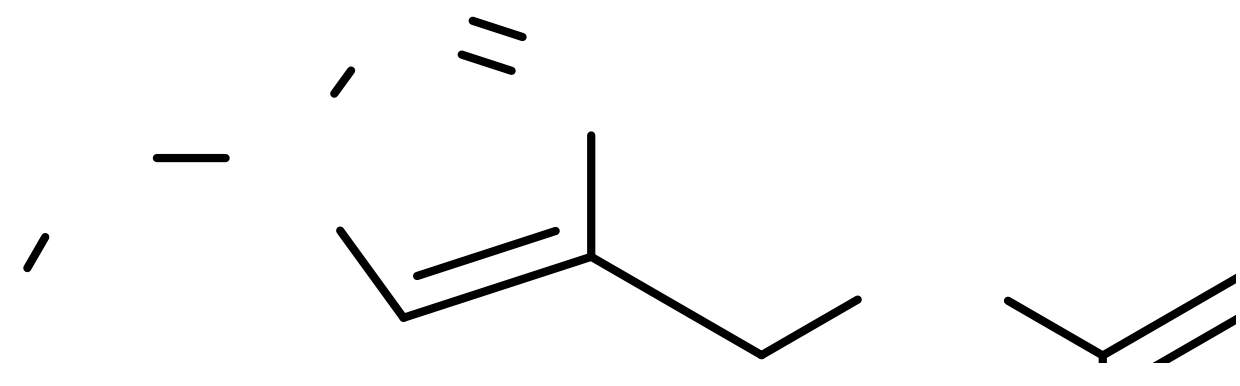

\subsection{CA IX inhibitors as radiopharmaceuticals -2 .}

Molecular Insight Pharmaceuticals, Inc. is a company that focuses on the discovery and development of radiopharmaceuticals for use in oncology. The company has filed a world patent application describing the design, synthesis and administration of CA inhibitors as radiopharmaceuticals for use in cancer diagnostic imaging and as a radiotherapy. ${ }^{36}$ The basis of this patent application is to exploit the selective expression of CA IX on hypoxic tumor cell surfaces by targeting with CA IX inhibitors that have radiopharmaceutical properties. The radiopharmaceutical compounds or complexes were designed to include the common aromatic/heteroaromatic sulfonamide for CA inhibition, and either a radionuclide metalchelate complex (type 135) or radioactive halogen (type 136). 


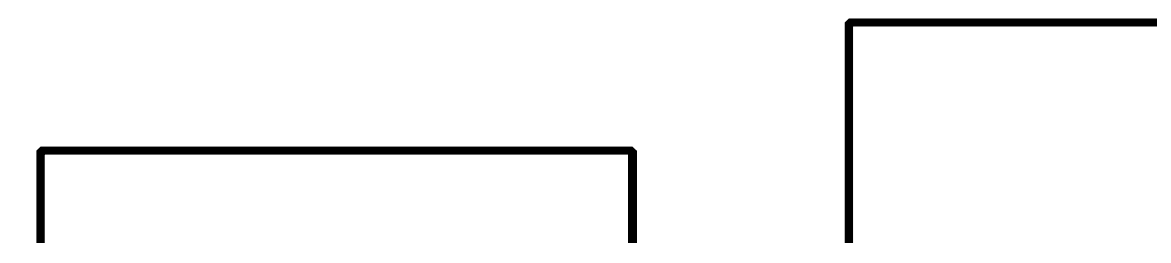

Exemplary radionuclide metals are technetium (as ${ }^{99 \mathrm{~m}} \mathrm{Tc}$ ) and rhenium (as ${ }^{185,186,188} \mathrm{Re}$ ) as in example compound 137, while a typical radioactive halogen was ${ }^{123} \mathrm{I}$ or ${ }^{131} \mathrm{I}$ as in example compound 138. The synthesis of compounds of type 137 typically involved the covalent reaction of a metal-chelate complex with an aromatic/heteroaromatic sulfonamide scaffold using straight forward chemistry such as amide coupling or 1,2,3-triazole formation using click chemistry. The synthesis of compounds of type $\mathbf{1 3 8}$ typically involved the reaction of trimethylstannane precursors with $\left[{ }^{123} \mathrm{I}\right] \mathrm{NaI}$ or $\left[{ }^{131} \mathrm{I}\right] \mathrm{NaI}$ to incorporate radioactive iodine.

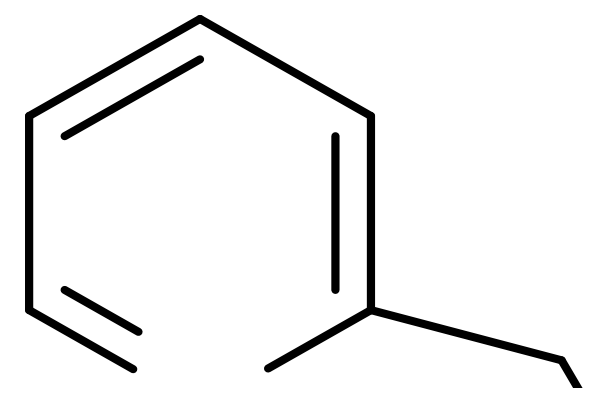

The patent application also describes the administration of these compounds to mammals by injection (intravenous, intramuscular or subcutaneous) as well as a product kit, with the kit containing a radiopharmaceutical and other components for end-use in a clinical setting. This application also discusses the design of membrane-impermeant CA inhibitors as an approach to selectively targeting CA IX over cytosolic CAs in vivo, most notably over CA II. The design approach taken towards membrane impermeable inhibitors was to prepare either bulky or positively or negatively charged inhibitors. 
Compounds were evaluated for inhibition of the esterase activity of CA IX. This assay spectrophotometrically monitors the hydrolysis of $p$-nitrophenylacetate to $p$-nitrophenolate in the presence of a test inhibitor compound. A single point test compound concentration of 1 $\mu \mathrm{M}$ was reported, with acetazolamide as the control CA inhibitor. Most compounds inhibited the CA IX esterase activity by $100 \%$, as did acetazolamide at $1 \mu \mathrm{M}$. This application does not present in vivo pharmacological studies or membrane permeability assays to support the claims regarding the therapeutic effects of these compounds, their ability to selectively target CA IX or their radiopharmaceutical properties.

\section{Summary}

In summary, the 2007-2009 patent application activity surrounding therapeutic applications of small molecule CA inhibitors in oncology have been filed by both small-mid sized pharmaceutical companies and academia, with Big Pharma applicants noticeably absent. These applications incorporate either classical chemotherapeutic or radiotherpeutic end use for cancer. The small molecules all contain a zinc binding function - sulfonamide or sulfamate - to ensure CA active site recognition and inhibition, while novelty of structure is found elsewhere in the molecules. A variety of different in vitro enzyme assays were employed to study the binding interactions with CA enzymes, including the $\mathrm{CO}_{2}$ hydration assay method, the CA-esterase activity assay, the fluorescence based displacement assay, ITC and a fluorescent thermal shift assay. There were no in vivo results presented to confirm the therapeutic claims regarding the effect of these inhibitors in oncology.

\section{Expert opinion}

There is an immense sense of urgency for the development of new cancer therapies owing to the 1.5 million individuals diagnosed with and the 500,000 who die of cancer each year. ${ }^{37}$ 
New molecular entities and biologic licence applications approved by the US Food and Drug Administrations Center for Drug Evaluation and Research associated with oncology are desperately needed, with just five new cancer related approvals in $2010 .^{38}$ We are now in an exciting era in which there is great potential to develop rational, hypothesis-driven, mechanism-based molecular therapeutics for cancer. ${ }^{39}$ CAs haves a brilliant track record as a model enzyme for countless studies across numerous scientific disciplines. ${ }^{3}$ It has been shown recently that cancer-associated CAs (isozymes IX an XII) are a pivotal response for hypoxic cancer cell survival and proliferation. ${ }^{9}$ Selective inhibition of CA IX and XII to disrupt the delicate CA-mediated $\mathrm{pH}$ homeostasis within the solid tumor microenvironment (necessary for cell viability) may provide for the rare but rewarding ability to discriminate cancer cells from healthy cells, an attractive attribute that is deficient in most existing cytotoxic chemotherapies. The credit for major discoveries regarding CA IX rests with the tenacity and persistence of several academic research groups over the past two decades, most notably Supuran's, at the University of Florence, and Pastorekova's at the Institute of Virology, Slovak Academy of Sciences. CAs potential as a therapeutic target in oncology has however been validated only recently ${ }^{9,13}$ and CAs now represent a mechanistically novel scientific opportunity with enormous potential leading to first-in-class therapeutics for hypoxic tumors.

The period for this review is likely premature for the most interesting and substantial breakthrough patents in relation to the role of CA as a cancer therapeutic target. The field has until recently lacked appropriate CA-relevant cell and animal models of tumor hypoxia for the evaluation of compounds, leaving the cause-effect relationship of CA inhibitors in cancer not able to be attributed to CA inhibition without doubt, however this cause-effect connection is assumed in the patent applications reviewed here. Verification of the ability of small molecules to modulate CA activity as a cancer therapy or as a diagnostic and/or prognostic 
tool will be facilitated by the development of specific inhibitors for CA IX and XII in the in vivo setting. This will lead to a greater understanding of the role of CAs in tumour biology and together with the recently available cell- and animal-based models of CAs in $\mathrm{pH}_{\mathrm{i}}$ regulation and tumour hypoxia will prove critical for the evaluation of the future therapeutic impact of CA inhibition.

CA IX antibodies have known demonstrated potential as a radiopharmaceuticals and this has now been followed up with small molecule CA IX inhibitors in place of CA IX antibodies. Patent applications were filed by both by both Siemens Medical Solutions USA, Inc. (see $\mathbf{2 . 4}$ -1) and Molecular Insight Pharmaceuticals, Inc. (see 2.5) to this effect. Small molecules may offer a number of advantages over antibodies, including cost and ease of manufacture as well as improved biopharmaceutical ADME (absorption, distribution, metabolism and excretion) and DMPK (drug metabolism and pharmacokinetics) properties. The patent applications in the radiopharmaceutical area are thus quite interesting and the followup on these patents including the in vivo and/or clinical performance of these compounds is earnestly awaited.

The patent applications utilise a cross section of in vitro CA assay methods, however only one of these methods, the $\mathrm{CO}_{2}$ hydration method, confirms CA inhibition activity. Other assay methods are technically easier to carry out, however they provide the field with inconsistencies. Drug discovery around CA inhibition will necessitate at some point that the endogenous activity of the CA enzyme be evaluated in the presence of an inhibitor, and a move away from indirect assays to the $\mathrm{CO}_{2}$ hydration method is warranted.

Given the active site similarities of the 12 catalytically active human CAs, ${ }^{3}$ CAs are a challenging drug target. It would be useful for researchers to have comprehensive CA 
inhibition data across all CA isozymes. At the very least small molecule inhibitors should be assessed at the physiologically dominant CA I and II as well as the cancer-associated CA IX and XII. To transform a small molecule inhibitor into a medicine that can be used in people requires conquering a spectrum of challenges and the physicochemical and toxicological properties that make a compound drug-like and safe must be considered alongside the goal to deliver compounds with desirable enzyme activity and selectivity. The CA field should commence with earnest to address drug like ADME and DMPK properties at the outset, in particular the evaluation of general toxicity needs to become routine in order to identify this potential liability early. There is currently a heavy reliance on in vitro enzyme assay results, yet a compounds in vivo or clinical performance cannot be inferred from this. Finally, the CA active sites are extremely tolerant to diverse and variable structural characteristics within the tail moiety of small molecule inhibitors. Provided there is a suitable zinc binding group such as sulfonamide or sulfamate, inhibitor tail groups may vary considerable in size, shape, charge, polarity, hydrophobicity etc. and yet still give nM inhibition. This minimal structural requirement in principle could permit an inhibitor design and development strategy that focuses less on optimizing structure-activity relationships (SAR) and more on finetuning the biopharmaceutical and toxicological properties of the inhibitor through structural manipulations. This attention towards optimizing structure-property relationships (SPR) may more rapidly guide this field towards drug discovery.

\section{Article highlights box}

- It has been shown recently that cancer-associated carbonic anhydrases (isozymes IX an XII) are a pivotal prosurvival enzymes during tumour hypoxia by regulating intracellular $\mathrm{pH}$. 
- Using shRNA gene silencing technology in a transfected LS174T colorectal cancer xenograft models ca9 and ca12 gene silencing caused a dramatic $85 \%$ reduction in tumor volume.

- The CA field has until recently lacked CA-relevant cell and animal models of tumor hypoxia for the therapeutic evaluation of small molecules. Verification of the ability of small molecules to modulate CA activity as a cancer therapy is however now possible with these models.

- CA inhibition is a new mechanism of action in oncology and small molecule inhibitors are under development as both classical chemotherapies for treatment and as radiopharmaceuticals for diagnostics and monitoring.

\section{References}

[1] Scozzafava A, Mastrolorenzo A, Supuran CT. Carbonic anhydrase inhibitors and activators and their use in therapy. Expert Opin Ther Patents 2006; 16: 1627-64.

[2] Krishnamurthy VM, Kaufman GK, Urbach AR, Gitlin I, Gudiksen KL, Weibel DB, Whitesides GM. Carbonic Anhydrase as a Model for Biophysical and Physical-Organic Studies of Proteins and Proteiñ Ligand Binding. Chem Rev 2008; 108: $94 \tilde{6}$-1051.

[3] Supuran CT. Carbonic anhydrases: novel therapeutic applications for inhibitors and activators Nature Rev Drug Disc 2008; 7: 168-81.

•• A recent comprehensive review covering therapeutic applications of CA inhibitors.

[4] Brahimi-Horn MC, Pouyssegur J. HIF at a glance. J Cell Sci 2009; 122: 1055-57.

[5] Wykoff CC, Beasley NJ,Watson PH, Turner KJ, Pastorek J, Sibtain A, Wilson GD, Turley H, Talks KL, Maxwell PH, Pugh CW, Ratcliffe PJ, Harris AL. Hypoxia-inducible expression of tumor-associated carbonic anhydrases. Cancer Res 2000: 60; 7075-83. 
[6] Potter CPS, AL Harris AL. Diagnostic, prognostic and therapeutic implications of carbonic anhydrases in cancer. Br J Cancer 2003; 89: 2-7.

[7] Kaluz S, Kaluzova M, Liao S-Y, Lerman M, Stanbridge EJ. Transcriptional control of the tumor- and hypoxia-marker carbonic anhydrase 9: A one transcription factor (HIF-1) show? Biochim Biophys Acta Reviews on Cancer 2009; 1795: 162-72.

[8] Opavský R, Pastoreková S, Zelník V, Gibadulinová A, Stanbridge EJ, Závada J, Kettmann R, Pastorek J. Human MN/CA9 gene, a novel member of the carbonic anhydrase family: Structure and exon to protein domain relationships. Genomics 1996; 33: 480-87.

[9] Chiche J, Ilc K, Laferrière J, Trottier E, Dayan F, Mazure NM, Brahimi-Horn MC, Pouysségur J. Hypoxia-inducible carbonic anhydrase IX and XII promote tumor cell growth by counteracting acidosis through the regulation of the intracellular pH. Cancer Res 2009; 69: 358-68.

•• This paper provides the evidence that has validated CA IX and XII as a drug target in hypoxic tumours.

[10] Swietach P, Vaughan-Jones RD, Harris AL. Regulation of tumor $\mathrm{pH}$ and the role of carbonic anhydrase 9. Cancer Metastasis Rev 2007; 26: 299-310.

[11] Martinez-Zaguilan R, Seftor EA, Seftor RE, Chu YW, Gillies RJ, Hendrix MJ. Acidic pH enhances the invasive behavior of human melanoma cells. Clin Exp Metastasis 1996;14: 176-86.

[12] Swietach P, Wigfield S, Cobden P, Supuran CT, Harris AL, Vaughan-Jones RD. Tumorassociated carbonic anhydrase 9 spatially coordinates intracellular $\mathrm{pH}$ in three-dimensional multicellular growths. J Biol Chem 2008; 283: 20473-83.

[13] Swietach P, Patiar S, Supuran CT, Harris AL, Vaughan-Jones RD. The Role of Carbonic Anhydrase 9 in Regulating Extracellular and Intracellular pH in Three-dimensional Tumor Cell Growths. J Biol Chem 2009; 284: 20299-20310. 
[14] Svastova E, Hulikova A, Rafajova M, Zat'ovicova M, Gibadulinova A, Casini A, Cecchi A, Scozzafava A, Supuran CT, Pastorek J, Pastorekova S. Hypoxia activates the capacity of tumor-associated carbonic anhydrase IX to acidify extracellular pH. FEBS Letters 2004; 577: 439-45.

[15] Divgi CR, Bander NH, Scott AM, O’Donoghue JA, Sgouros G, Welt S, Finn RD, Morrissey F, Capitelli P, Williams JM, Deland D, Nakhre A, Oosterwijk E, Gulec S, Graham MC, Larson SM, Old LJPhase I/II radioimmunotherapy trial with iodine-131-labeled monoclonal antibody G250 in metastatic renal cell carcinoma. Clin Cancer Res 1998; 4: $2729-39$.

- Describing a radioimmunotherapy trial with a CA IX monoclonal antibody.

[16] Grabmaier K, Vissers JL, De Weijert MC, Oosterwijk-Wakka JC, van Bokhoven A, Brakenhoff RH, Noessner E, Mulders PA, Merkx G, Figdor, CG, Adema, GJ, Oosterwijk E. Molecular cloning and immunogenicity of renal cell carcinoma-associated antigen G250. Int J Cancer 2000; 85: 865-870.

- G250 antigen first characterised as CA IX.

[17] Lopez M, Bornaghi LF, Innocenti A, Vullo D, Charman SA, Supuran CT, Poulsen S-A. Sulfonamide linked neoglycoconjugates - a new class of inhibitors for cancer-associated carbonic anhydrases. J Med Chem 2010: published online 4 March 2010, doi: 10.1021/jm901888x.

[18] Lopez M, Paul B, Hofmann A, Morizzi J, Wu Q, Charman SA, Innocenti A, Vullo D, Supuran CT, Poulsen S-A. S-glycosyl primary sulfonamides - A new structural class for selective inhibition of cancer-associated carbonic anhydrases. J Med Chem 2009; 52: 642132.

- Paper describing structure-property relationships of small molecule inhibitors targetting CA IX and XII. 
[19] Supuran C, Scozzafava A, Pastorekova S, Pastorek J. Sulfonamide carbonic anhydrase IX-specific inhibitors for the treatment of preneoplastic and neoplastic diseases. WO048544 (2004).

[20] Winum J-Y, Poulsen S-A, Supuran CT. Therapeutic applications of glycosidic carbonic anhydrase inhibitors. Med Res Rev 2009; 29: 419-35.

[21] Winum J-Y, Rami M, Scozzafava A, Montero J-L, Supuran CT. Carbonic anhydrase IX: A new druggable target for the design of antitumor agents. Med Res Rev 2008; 28: 445-63.

[22] Stiti M, Cecchi A, Rami M, Abdaoui M, Barragan-Montero V, Scozzafava A, Guari Y, Winum J-Y, Supuran CT. Carbonic anhydrase inhibitor coated gold nanoparticles selectively inhibit the tumor-associated isoform IX over the cytosolic isozymes I and II. J Am Chem Soc 2008; 130: 16130-1.

[23] Nicox S.A. Nitrate esters of carbonic anhydrase inhibitors. WO071421 (2008)

[24] Solvay Pharmaceuticals GMBH. Novel sulfamate compounds for medical use. WO050252 (2009)

[25] Maryanoff BE. Pharmaceutical "gold” from neurostabilizing agents: Topiramate and successor molecules. J Med Chem 2009; 52: 3431-40.

[26] Casini A, Antel J, Abbate F, Scozzafava A, David S, Waldeck H, Schafer S, Supuran CT. Carbonic anhydrase inhibitors: SAR and X-ray crystallographic study for the interaction of sugar sulfamates/sulfamides with isozymes I, II and IV. Bioorg Med Chem Lett 2003; 13: 841-45.

[27] Winum J-Y, Scozzafava A, Montero J-L, Supuran CT. Sulfamates and their therapeutic potential. Med Res Rev 2005; 25: 186-228.

[28] Okada M, Iwashita S, Koizumi N. Efficient general method for sulfamoylation of a hydroxyl group. Tetrahedron Lett 2000; 41: 7047-51. 
[29] Biotechnologijos Institutas. Benzimidazo[1,2,-c][1,2,3]thiadiazol-7-sulfonamides as CA inhibitors. WO016288 (2008).

[30] Dudutiene V, Baranauskiene L, Matulis D. Benzimidazo[1,2-c][1,2,3]thiadiazole-7sulfonamides as inhibitors of carbonic anhydrase. Bioorg Med Chem Lett 17; 2007: 3335-38. [31] Siemens Medical Solutions USA, Inc. The development of molecular imaging probes for carbonic anhydrase-IX using click chemistry. WO124703 (2008).

[32] Scozzafava A, Menabuoni L, Mincione L, Briganti F, Mincione G, Supuran CT. Carbonic anhydrase inhibitors. Synthesis of water-soluble, topically effective, intraocular pressure-lowering aromatic/heterocyclic sulfonamides containing cationic or anionic moieties: Is the tail more important than the ring? J Med Chem 1999: 42, 2641-50.

[33] Wilkinson BL, Bornaghi LF, Houston TA, Innocenti A, Supuran CT, Poulsen S-A. A novel class of carbonic anhydrase inhibitors: glycoconjugate benzene sulfonamides prepared by “click-tailing”. J Med Chem 2006; 49: 6539-48.

[34] Poulsen S-A, Bornaghi LF. Fragment-based drug discovery of carbonic anhydrase II inhibitors by dynamic combinatorial chemistry utilising alkene cross metathesis. Bioorg Med Chem 2006; 14: 3275-3284.

[35] Supuran CT, Scozzafava A, Conway J. Carbonic Anhydrase: Its Inhibitors and Activators. Florida: CRC Press, 2004.

[36] Molecular Insight Pharmaceuticals, Inc. Inhibitors of carbonic anhydrase IX. WO089383 (2009).

[37] Druker BJ. Perspectives on the development of imatinib and the future of cancer research. Nature Medicine 2009; 15: 1149-52.

[38] Hughes B. 2009 FDA drug approvals. Nature Rev Drug Disc 2010; 9: 89-92.

[39] Collins I, Workman P. New approaches to molecular cancer therapeutics. Nature Chem Biol 2006; 2: 689-700. 


\section{Figure and Scheme Legends.}

Scheme 1. Carbonic anhydrases catalyze the reversible hydration of carbon dioxide to give bicarbonate anion and a proton.

Scheme 2. Catalytic cycle of CAs. The active site $\mathrm{Zn}^{2+}$ is coordinated to three histidine residues and the substrate $\mathrm{H}_{2} \mathrm{O}$ molecule. The $\mathrm{Zn}^{2+}$ coordinated water is deprotonated to generate the strongly basic hydroxide anion which is the reactive species in the hydration of $\mathrm{CO}_{2}$ converting it to $\mathrm{HCO}_{3}^{-}$.

Figure 1. Schematic of normoxic and hypoxic cells demonstrating the pro-survival role of CAs in hypoxic tumours through $\mathrm{pH}_{\mathrm{i}}$ regulation.

Figure 2. Bicarbonate substrate and sulfonamide inhibitor $\left(\mathrm{R}-\mathrm{SO}_{2} \mathrm{NH}_{2}\right)$ bound in the active site of CAs.

Figure 3. Clinically used aromatic or heteroaromatic sulfonamide inhibitors of CAs. All are representatives of the $\mathrm{R}-\mathrm{SO}_{2} \mathrm{NH}_{2}$ pharmacophore.

Scheme 3. Synthesis by $\mathrm{Cu}(\mathrm{I})$ catalyzed 1,3-dipolar cycloaddition reaction (1,3-DCR) of azide with alkyne CA inhibitor scaffold 127.

Scheme 4. Synthesis of a triazole CA inhibitor (compound 134) as a PET ligands for imaging CA IX expressing tumors. $\mathrm{P}=$ protecting group, $\mathrm{LG}=$ leaving group. 